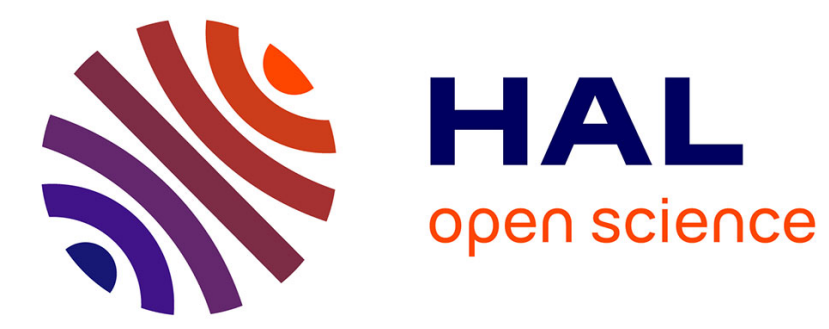

\title{
Coherent scattering by one reptating chain
}

\author{
P.-G. de Gennes
}

\section{To cite this version:}

P.-G. de Gennes. Coherent scattering by one reptating chain. Journal de Physique, 1981, 42 (5), pp.735-740. 10.1051/jphys:01981004205073500 . jpa-00209059

\section{HAL Id: jpa-00209059 https://hal.science/jpa-00209059}

Submitted on 1 Jan 1981

HAL is a multi-disciplinary open access archive for the deposit and dissemination of scientific research documents, whether they are published or not. The documents may come from teaching and research institutions in France or abroad, or from public or private research centers.
L'archive ouverte pluridisciplinaire HAL, est destinée au dépôt et à la diffusion de documents scientifiques de niveau recherche, publiés ou non, émanant des établissements d'enseignement et de recherche français ou étrangers, des laboratoires publics ou privés. 
Classification

Physics Abstracts

$61.40 \mathrm{~K}$

\title{
Coherent scattering by one reptating chain
}

\author{
P. G. De Gennes \\ Physique de la Matière Condensée, Collège de France, 75231 Paris Cedex 05, France
}

(Reçu le 21 novembre 1980, accepté le 12 janvier 1981)

\begin{abstract}
Résumé. - On analyse la structure de la fonction de diffusion cohérente $S_{q}(t)$ pour une chaîne polymérique deutérée se déplaçant dans un fondu de chaînes enchevêtrées, chimiquement identiques mais non deutérées. Le domaine intéressant correspond à des longueurs d'onde $2 \pi / q$ plus petites que la taille globale de la chaîne $\left(R_{0}\right)$ mais plus grandes que la distance $D$ entre points d'enchevêtrement. Dans ce domaine, nous sommes conduits à une forte autocritique : les résultats sont beaucoup plus complexes que nous ne l'avions imaginé dans un travail antérieur [1]. La fonction $S_{q}(t)$ se sépare en :a) une partie $S_{q}^{1}(t)$ qui décrit des fluctuations locales dans le tube, et qui relaxe relativement vite; $b$ ) une partie $S_{q}^{c}(t)$ qui est de plus grande amplitude, et qui ne décroît que très lentement (avec pour temps caractéristique le temps de reptation globale $T_{\text {rep }}$ ). Dans la référence [1] nous avions postulé une seule fréquence caractéristique $\left(1 / \tau_{q} \sim q^{6}\right)$. Il s'avère que cet $1 / \tau_{q}$ est seulement la moyenne pondérée des deux fréquences physiques associées à $S^{1}$ et $S^{c}$.

Abstract. - We discuss the time dependent correlation functions $S_{q}(t)$ for one deuterated polymer chain, moving inside an entangled melt of chemically identical, protonated, chains. The region of interest corresponds to wavevectors $q$ such that $D^{-1} \gg q \gg R_{0}^{-1}$ where $D$ is the tube diameter and $R_{0}$ the overall chain size. In this regime the results disagree strongly with our earlier prediction [1]. We find that the function $S_{q}(t)$ breaks up into two parts. One part $\left[S_{q}^{1}(t)\right]$ describes the local fluctuations of « kinks » inside the tube and relaxes relatively fast (characteristic time $\left.\sim q^{-4}\right)$. The other part $\left[S_{q}^{c}(t)\right]$, with much larger amplitude, describes a slow, global creep of the chain inside its tube, associated with the tube diffusivity $D_{t}$. The characteristic time for this creep process is independent of $q$, and is the overall reptation time $T_{\text {rep. }}$. In reference [1] $a$ single characteristic rate $1 / \tau_{q} \sim q^{6}$ was constructed for each $q$ vector : this turns out to be the weighted average of the two physical relaxation rates.
\end{abstract}

1. Introduction. - The weakly inelastic scattering of neutrons by flexible polymers has been studied in various melts [2]. Depending on the details of the situation (protonated versus deuterated species) one can measure either an incoherent scattering function

$$
S_{q}^{(\mathbf{i})}(t)=\sum_{n}\left\langle\exp \left[i \mathbf{q} \cdot\left(\mathbf{r}_{n}(t)-\mathbf{r}_{n}(0)\right)\right]\right\rangle
$$

or a coherent scattering function

$$
S_{q}(t) \doteq \sum_{n, m}\left\langle\exp \left[i \mathbf{i q} \cdot\left(\mathbf{r}_{m}(t)-\mathbf{r}_{n}(0)\right)\right]\right\rangle .
$$

In equation (1.1) each scattering atom $(n)$ contributes separately, while in equation (1.2) all pairs of scattering atoms $(n, m)$ are involved. The brackets represent thermal averages. The wavevector $q$ is related to the scattering angle by the usual formula. Finally the time variable $t$ is related to the energy transfer $\hbar \omega$ (between the neutron and the scattering system). The Fourier transform :

$$
S_{q}(\omega)=\frac{1}{2 \pi} \int \mathrm{d} t S_{q}(t) \mathrm{e}^{i \omega t}
$$

gives the scattering intensity for given $q$ and $\omega$ [3]. With the most recent spin echo techniques [4] one can in fact measure $S_{q}(t)$ directly, and reach times as long as $10^{-8}$ seconds.

Even with these sophisticated means, most of the neutron experiments deal with ranges of $q$ and $\omega$ which are somewhat too large to reach universal laws. However, the scattering function $S_{q}(t)$ is of more general interest $: a$ ) it can be measured by photon beat methods $\left({ }^{1}\right)$ at very low $\left.q ; b\right)$ from a theoretical standpoint, $S_{q}(t)$ contains essential information on the dynamics of the scattering objects.

( $\left.{ }^{1}\right)$ For an introduction to these methods as applied to polymers, see for instance, P. G. de Gennes, "Scaling concepts in polymer physics" (Cornell Univ. Press) 1979, p. 177. 
For polymer chains, with $N$ beads $(N \gg 1)$ and an end to end (r.m.s.) distance $R_{0}=N^{1 / 2} a$, the regime

$$
\frac{1}{a} \gg q \gg \frac{1}{R_{0}}
$$

is most directly interesting : here we probe the inner modes of the chain, but the inequality $q a \ll 1$ ensures that the form of $S_{q}(t)$ is relatively universal (independent of the detailed chemical structure of the monomers).

For a single, ideal, Rouse chain [5] in solution, both the coherent and incoherent scattering functions have been calculated long ago [6]. Backflow effects have been incorporated later [7]. However, the most practical case is different : it corresponds to a melt of entangled chains. Here the dominant polymer motions are "reptations" in a tube [8]. The mechanical consequences of the reptation picture have been worked out by Doi and Edwards [9], and are in reasonable agreement with experiment.

The incoherent scattering properties of a reptating chain were already described qualitatively in reference [8]. The coherent scattering properties are more delicate. In a recent paper [1] the present author attempted to estimate the characteristic frequency range $1 / \tau_{q}$ associated with one given $q$. Unfortunately, this attempt was a failure ! We discovered this first when discussing a problem of polymer welding [10] where very local motions occur near an interface (i.e. $q R_{0} \gg 1$ ). The results of a relatively transparent analysis for welding turned out to be qualitatively different from the prediction of reference [1]. We then decided to construct the coherent form factor in more detail, for the simplest case available. This corresponds to one deuterated chain moving in a melt of other chains which are chemically equivalent but isotopically different. Experiments of this type have been recently carried out by the spin echo technique [4]. Unfortunately, as mentioned above, they deal mainly with $q$ values which are too high : $q D \widetilde{>} 1$ where $D=N_{\mathrm{e}}^{1 / 2} a$ is the distance between entanglements ( $N_{\mathrm{e}}$ being the corresponding number of monomers). At $q D>1$ the entanglements become irrelevant and the simple Rouse behaviour of reference [6] should be recovered. In the present paper we are concerned with the opposite limit

$$
D^{-1}>q>R_{0}^{-1}
$$

where entanglements are dominant. The ratio $R_{0} / D \sim\left(N / N_{\mathrm{e}}\right)^{1 / 2}$ is of order 10 in favourable cases : this means that the range of $q$ values allowed by the two inequalities (1.5) is not very large. However, to reach relatively simple results, it is clearly this limit which must be studied first. In section 2 we describe the main qualitative features of the coherent scattering function, and show that two distinct time scales are involved. In section 3 we give a more detailed calculation of the short time scales. In section 4 we compare the results of the present paper with those of reference [1].

2. The two basic processes. - 2.1 LoCAL REPTATION. - Let us consider first a sequence of times $t$ shorter than the Rouse time $T_{\text {ro }}=N^{2} W^{-1}$, where $W$ is a microscopic jump frequency. In the language of reference [8], this means that the "kinks " along our chain diffuse locally, but are not influenced by special effects at both ends of the chain. We call this regime local reptation. It is associated with internal dilations, or contractions of the chain inside a fixed tube.

The state of affairs at the end of this stage is represented on figure 1 : starting from a given conformation of the chain $(1 a)$ we reach a smeared density profile $(1 b)$ where the chain is still in the same tube, but where there is a certain uncertainty in the position of each monomer. The resulting value of $S_{q}(t)$ shall be called $\bar{S}_{q}$. It is independent of $t$ (since the smeared profile is time independent) and it is not much smaller than $S_{q}(0)$.

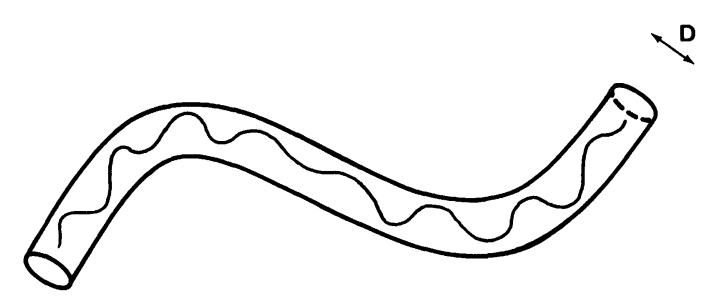

(a)

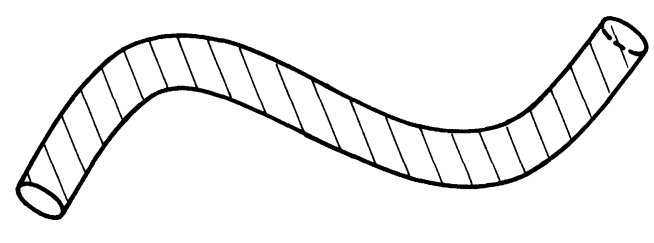

(b)

Fig. 1. - Local reptation processes during a time $t$ smaller than the Rouse time. $a$ ) The chain starts from a given conformation and is trapped in a certain tube. $b$ ) At a later time $t$ the chain is still caught in the same tube portion, but its probability distribution is smeared out over a diameter $D$.

This may seem surprising, since any given monomer $(m)$ drifts randomly along the tube, and moves by relatively large distances $s \sim t^{1 / 4}$ in a time $t$, as was shown in reference [8]. If the motions of the monomers were independent at large $t$, we could write

$$
\begin{aligned}
\bar{S}_{q} & =\lim _{t \rightarrow \infty} \sum_{n m}\left\langle\mathrm{e}^{i \mathbf{q} \cdot \mathbf{r}_{m}(t)} \mathrm{e}^{-i \mathbf{q} \cdot \mathbf{r}_{n}(0)}\right\rangle \\
& =\sum_{n m}\left\langle\mathrm{e}^{i \mathbf{q} \cdot \mathbf{r}_{m}(t)}\right\rangle\left\langle\mathrm{e}^{-i \mathbf{q} \cdot \mathbf{r}_{n}(0)}\right\rangle .
\end{aligned}
$$


This in turn would imply

$$
\bar{S}_{q}=0 \quad\left(q R_{0} \gg 1\right)
$$

since the distribution of $\mathbf{r}_{n}$ (or $\mathbf{r}_{m}$ ) at any instant is spread over distances $R_{0} \gg q^{-1}$. However equations $(2.1,2)$ are wrong, because the monomers remain correlated and achieve, at high $t$, a constant density along all the tube length.

Although monomer $m$ may have drifted far during time $t$, it is still in the tube and surrounded by other monomers $(n)$. The scattering function $\bar{S}(q)$ is controlled by the number of pairs $(n m)$ for which $\left|r_{m}(t)-r_{n}(0)\right|$ is smaller than $1 / q$.

a) If smearing is ignored, the number of active neighbours $(n)$ of $(m)$, is given by ideal chain statistics, and is $g_{q} \sim 1 /(q a)^{2}$ (the local analog of $\left.N=\left(R_{0} / a\right)^{2}\right)$. Including the exact coefficients (computed long ago by Debye), this would give

$$
\frac{1}{N} \bar{S}_{q}=\frac{12}{q^{2} a^{2}}=\frac{1}{N} S_{q}(0) \quad(q D \rightarrow 0) .
$$

b) If we include smearing, we must make the convolution of the ideal chain distribution for $\mathbf{r}_{n}$ by some smearing function of width $\sim D$. For instance we could take a gaussian, and write

$$
\bar{S}_{q}=S_{q}(0) \exp -\alpha^{2} D^{2} q^{2}
$$

where $\alpha$ is a numerical constant. Since we want $q D \ll 1$ we can write

$$
\bar{S}_{q}=S_{q}(0)\left(1-\alpha^{2} q^{2} D^{2}\right) .
$$

Equation (2.4) is dependent on a specific model for the tube, but equation (2.5) is not : it shows that $\bar{S}_{q}$ is only slightly smaller than $S_{q}(D)$ : the function $S_{q}(t)$ does not decrease much by local reptation processes. In section 3 we analyse the details of this relaxation. We give a precise value for the coefficient $\alpha$ in (2.4) and we show that the characteristic time for relaxation is

$$
\tau_{\mathrm{loc}}(q) \cong W^{-1}(q a)^{-4}
$$

where $W$ is a microscopic frequency (independent of $N$ and of $N_{\mathrm{e}}$ ) defined as in reference [6].

2.2 CREEP. - Let us now consider intervals $t$ larger than the Rouse time : in such an interval, the " kinks " have reached equilibrium inside the chain. There remains, however, a very slow, global, reptation of the chain, associated with a «tube diffusion constant » $[8] D_{\mathrm{t}}$.

In this regime, the chain moves as a whole, and progressively creeps out of its original tube. The time required for complete exit is the reptation time $T_{\text {rep}}$, and is defined by

$$
D_{\mathrm{t}} T_{\text {rep }}=\pi^{-2} L^{2} .
$$

Here $L$ is the original tube length, and is

$$
L=\frac{N}{N_{\mathrm{e}}} D=N a^{2} / D \cong N a N_{\mathrm{e}}^{-1 / 2} .
$$

Equation (2.8) may be understood through a division of the chain into subunits, each of $N_{\mathrm{e}}$ monomers and of linear dimensions $D$. We shall take it as our precise definition of $N_{\mathrm{e}}$ (or $D$ ).

Let us also recall the structure of the tube diffusion coefficient

$$
D_{\mathrm{t}}=\frac{1}{3} a^{2} W N^{-1}
$$

very similar to what we have for a free Rouse chain [5]. Combining $(2.7,8,9)$ we recover the classical features of the reptation time :

$$
T_{\text {rep }} \cong \tau_{0} N^{3} / N_{\mathrm{e}}
$$

Having these concepts in mind we can construct the structure of $S_{q}(t)$ for large times $\left(t \sim T_{\text {rep }}\right)$ and large $q$ vectors $\left(q R_{0} \gg 1\right)$. The principle is explained in figure 2. Using again the argument of section 2, we say that two monomers $(m)$ and $(n)$ contribute to $S_{q}(t)$ only if

$$
\left|\mathbf{r}_{m}(t)-\mathbf{r}_{n}(0)\right| \approx q^{-1}
$$

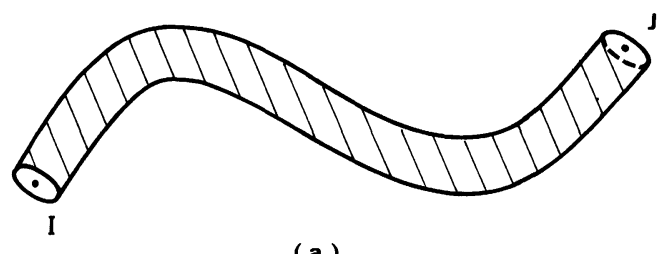

(a)

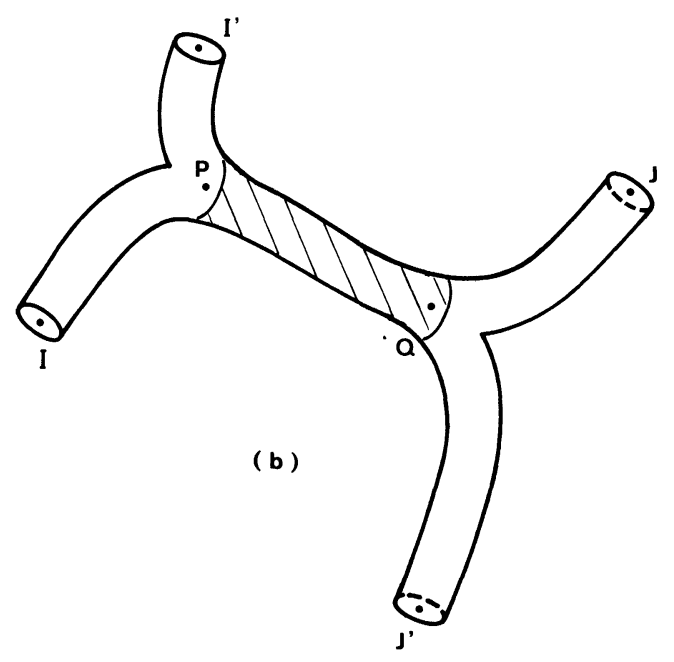

Fig. 2. - Calculation of the coherent scattering by one chain at times $t \sim T_{\text {rep }}$ a) Original tube $\left.\mathrm{IJ} ; b\right)$ at time $t$ a new tube $\mathrm{I}^{\prime} \mathrm{J}^{\prime}$ is occupied. At large wavector $q>R_{0}^{-1}$ the coherent scattering function $S_{q}(t)$ is dominated by the part which is common to both tubes. 
If, at time $t$, the monomer $m$ has drifted out of the original tube, it will be separated from all points inside the original tube by distances of order $R_{0}$ (much larger than $q^{-1}$ ). Thus the only pairs $m, n$ which contribute are those for which $\mathbf{r}_{m}(t)$ is still located in the original tube.

Repeating the argument of section (2.1), this gives

$$
S_{q}(t)=\bar{S}_{q} \frac{\widetilde{N}(t)}{N}
$$

where $\bar{S}_{q}$ is given in equation (2.5), while $\tilde{N}(t)$ is the number of monomers $(m)$ which are still trapped at time $t$ inside the original tube (formed at $t=0$ ). The result $(2.12)$ has already been derived by Doi and Edwards [9].

The factor $\tilde{N}(t) / N$ is familiar in reptation studies :

a) it controls the relaxation of the end to end vector of the chain [8];

$b)$ it controls the memory function for linear viscoelastic behaviour [9].

$\tilde{N}(t) / N$ was calculated first in section 5 of reference [8] :

$$
\frac{\tilde{N}(t)}{N}=\frac{8}{\pi^{2}} \sum_{n \text { odd }} n^{-2} \exp \left(-n^{2} t / T_{\text {rep }}\right)
$$

where $T_{\text {rep }}$ is defined explicitly through equations $(2.7,9)$.

The leading term of this expression at high times corresponds to $n=1$ and gives a simple exponential decay with time constant $T_{\text {rep }}$.

Thus we conclude that, in the creep regime, with $q R_{0} \gg 1$, the coherent scattering function has a decay rate $\left(1 / T_{\text {rep }}\right)$ which is independent of the wavevector. This is completely different from what is stated in reference [1].

3. Local reptation. - We discuss now in more detail the motions of a very long chain for times $t$ smaller than the overall Rouse time $N^{2} W^{-1}$.

In a first step we consider local reptation processes in a rectilinear tube. In a second step we map the results into the real situation, where the tube itself is randomly curved. We assume that the microscopic dynamics is the same for both situations.

3.1 Motions AlONG A Straight tUBe. - The central parameter here is the number density $v(s . t)$, defined as follows, $v(s) \mathrm{d} s$ is the number of monomers in an element of length $\mathrm{d} s$ along the tube. We must emphasize first that the average value of $v$ (which we call $\bar{v}$ ) is finite and constant in all the tube region (of length $L$ ) occupied by the chain.

$$
\bar{v}=\frac{N_{\mathrm{e}}}{D}=N_{\mathrm{e}}^{1 / 2} a^{-1} .
$$

This implies that our chain cannot be described as an entirely free Rouse chain : the latter would give $N$ monomers spread over a tube length $\sim R_{0}$ and give densities $N / R_{0}$ which are far too high. What we must do is to impose a tension at both ends of the chain. Then we can achieve an average stretch per monomer

$$
l=\frac{L}{N}=(\bar{v})^{-1}=a N_{\mathrm{e}}^{-1 / 2} .
$$

We are interested here in the fluctuations of the density $v$ and describe them in terms of a correlation function :

$$
\Sigma(s, t)=\langle v(s, t) v(0,0)\rangle .
$$

The Fourier transform of $\Sigma(s)$ is (apart from a normalization factor) the coherent scattering function for a straight tube, which we call $\Sigma_{p}(t)$

$$
\Sigma_{p}(t)=L \int \mathrm{d} s \mathrm{e}^{i p s} \Sigma(s, t) .
$$

The calculation of $\Sigma_{p}(t)$ for a Rouse chain under stretch was performed at the end of reference [6] :

$$
\Sigma_{p}(t)=\frac{N a^{2}}{3 l^{2}} \exp \left(-\Delta t p^{2}\right)
$$

where

$$
\Delta=W l^{2}=W a^{2} / N_{\mathrm{e}}
$$

is (in the language of reference [8]) the diffusion coefficient of the kinks. Note that the time for complete equilibration of the kinks over the whole chain is

$$
\frac{L^{2}}{\Delta}=\frac{N^{2}}{W}=T_{\text {ro }}
$$

and is identical to the Rouse time of a free chain (independent of $N_{\mathrm{e}}$ ).

Returning to the spatial properties along the tube, by inversion of (3.5) we arrive at the required result for a straight tube. Since the Fourier transform of a gaussian is a gaussian, we get :

$$
\Sigma(s t)=\bar{v}^{2}+\frac{\bar{v} N_{\mathrm{e}}}{3} \frac{1}{(4 \pi \Delta t)^{1 / 2}} \exp \left[-s^{2} /(4 \Delta t)\right] .
$$

3.2 Contorted TUBE. - We still call $s$ the curvilinear abscissa along the tube, and write a coarsegrained position for a monomer as $\mathbf{r}(s)$ (the size of the coarse-graining is the tube diameter $D$ ). Then we may transform from monomer indices $(n, m)$ to position indices $\left(s_{1}, s_{2}\right)$ :

$$
\begin{array}{rl}
S_{\hat{q}}(t)=\iint_{0}^{L} \mathrm{~d} s_{1} & \mathrm{~d} s_{2} \\
\times & \Sigma\left(s_{1}-s_{2}, t\right) \times \\
\times & \left\langle\exp i \mathbf{q} \cdot\left[\mathbf{r}\left(s_{1}\right)-\mathbf{r}\left(s_{2}\right)\right]\right\rangle .
\end{array}
$$


Because the tube is itself a gaussian chain we have $\left\langle\exp i q \cdot\left[\mathbf{r}\left(s_{1}\right)-\mathbf{r}\left(s_{2}\right)\right]\right\rangle=$

$$
\begin{aligned}
& =\exp -\frac{1}{2}\left\langle\left[\mathbf{q} \cdot\left(\mathbf{r}\left(s_{1}\right)-\mathbf{r}\left(s_{2}\right)\right)\right]^{2}\right\rangle \\
& =\exp -\frac{q^{2} a^{2}}{6 l}\left|s_{1}-s_{2}\right| \quad(3.10)
\end{aligned}
$$

because the interval $\left(s_{1}, s_{2}\right)$ contains $\frac{\left|s_{1}-s_{2}\right|}{l}$ monomers, each of r.m.s. size $a$. Ultimately equation $(3.9,10)$ give us the answer in the form

$$
\begin{array}{rl}
S_{q}(t)=\bar{v}^{2} & L l \frac{12}{q^{2} a^{2}}+\frac{N N_{\mathrm{e}}}{3} \frac{2}{(4 \pi \Delta t)^{1 / 2}} \times \\
\times \int_{0}^{\infty} \mathrm{d} s \exp \left[-\frac{q^{2} a^{2}}{6 l} s-\frac{s^{2}}{4 \Delta t}\right] .
\end{array}
$$

In our coarse-grained approximation the first term is simply $S_{q}(0)$ as defined in equation (2.3). However if we had included corrections of order $(q D)^{2}$ we should have obtained a slightly smaller first term, corresponding to $\bar{S}(q)$ (Eq. (2.5)). We can write down the correction explicitly by imposing that at $t=0$ the sum of both terms in equation (3.11) be exactly $S_{q}(0)$. The final result is :

$$
\begin{aligned}
& \bar{S}(q)=N\left[\frac{12}{q^{2} a^{2}}-\frac{N_{\mathrm{e}}}{3}\right]=\frac{N 12}{q^{2} a^{2}}\left(1-\frac{q^{2} D^{2}}{36}\right) \\
& S_{q}(t)=\bar{S}(q)+N N_{\mathrm{e}} f\left[q^{2} a^{2}(W t)^{1 / 2}\right] \\
& \quad\left(\frac{1}{D} \gg q \gg \frac{1}{R_{0}}\right) \\
& f(u)=\frac{1}{3 \pi^{1 / 2}} \int_{0}^{\infty} \mathrm{d} x \exp \left(-x u / 6-x^{2} / 4\right) .
\end{aligned}
$$

Equation (3.13) gives the decomposition of the coherent scattering function which was announced in section 3.1. The first term is time independent (for $\left.t \ll T_{\text {rep }}\right)$. The second term decays with a characteristic time $\tau_{\text {loc }}(q)$ proportional to $q^{-4}$, as quoted in equation (2.6). Notice that this decay is not at all exponential : from (3.11) we find that, for $u \ll 1$

$$
f(u)=\frac{1}{3}-\text { const. } u=\frac{1}{3}-\text { const } .\left(t / \tau_{\text {loc }}(q)\right)^{1 / 2}
$$

and for $u \gg 1$

$$
f(u)=\text { const. } u^{-1} \cong\left(\frac{\tau_{\mathrm{loc}}(q)}{t}\right)^{1 / 2}
$$

4. Conclusions. - For the wavevectors $q$ under consideration (defined in (1.5)), the overall coherent scattering function of one chain is the sum of two contributions with completely different structures

$$
S_{q}(t)=S_{q}^{1}(t)+S_{q}^{\mathrm{c}}(t)
$$

where 1 stands for "local " and c for « creep ». The local term is given by equation $(3.13,14)$

$$
S_{q}^{1}(t)=N N_{\mathrm{e}} f\left[q^{2} a^{2}(W t)^{1 / 2}\right]
$$

and its amplitude is measured by

$$
S_{q}^{1}(0)=\frac{N N_{\mathrm{e}}}{3} .
$$

The creep term is given by equations $(2.12,13)$ :

$$
\begin{aligned}
S_{q}^{\mathrm{c}}(t)=N\left(1-\frac{q^{2} D^{2}}{36}\right) \frac{12}{(q a)^{2}} \sum_{n \text { odd }} \frac{8 n^{-2}}{\pi^{2}} \times \\
\times \exp \left(-n^{2} t / T_{\text {rep }}\right) .
\end{aligned}
$$

Its amplitude is much larger

$$
S_{q}^{\mathrm{c}}(0)=\bar{S}_{q}=N \frac{12}{(q a)^{2}}\left(1-\frac{q^{2} D^{2}}{36}\right)
$$

Thus a complete discussion of coherent response functions must include two amplitudes and two relaxation times. The scaling conjecture of reference [1] was wrong because it assumed one single component.

Let us call $1 / \tau_{q}$ the weighted average of the two characteristic rates :

$$
\begin{aligned}
\frac{1}{\tau_{q}} & =\frac{S^{1}(0) W(q a)^{4}+S^{\mathrm{c}}(0) T_{\mathrm{rep}}^{-1}}{S^{1}(0)+S^{\mathrm{c}}(0)} \\
& \cong \frac{q^{2} D^{2}}{36} W(q a)^{4}+W N_{\mathrm{e}} N^{-3} \\
& \cong \frac{N_{\mathrm{e}}}{36} W(q a)^{6} .
\end{aligned}
$$

We see that $1 / \tau_{q}$ coincides with the characteristic rate which was constructed in reference [1] ! However this $1 / \tau(q)$ does not have much practical significance. For instance, if future neutron experiments are able to probe the $q$ region of interest $\left(D^{-1}>q>R_{0}^{-1}\right)$, they will give an inelastic intensity $S_{q}(\omega)$ which is a superposition of two terms

- the local term with a width

$$
\Delta \omega_{1}(q)=\text { const. } W(q a)^{4}
$$

- the creep term with a very small width

$$
\Delta \omega_{\mathrm{c}}(q)=1 / T_{\text {rep }},
$$

which in practice will be equivalent to a delta function (elastic scattering).

This distinction between a fast and a slow component will also be important for more complex situations, where we deal not with an isotropic mixture, but with a polymer blend, where interactions between the two components play a leading role. The consequences of the two component structure for these problems, involving spinodal decomposition, precipitate growth, etc., are currently under study [11]. 
Let us end up by a general remark : this paper was concerned with fluctuations of concentration (in an isotropic mixture) and with the related correlation functions. We wish to point out that similar problems will occur with other physical quantities. For instance we could look at the correlation function for shear stress $\sigma$ :

$$
\Gamma_{q}(t)=\left\langle\sigma_{-q}(0) \sigma_{q}(t)\right\rangle .
$$

Again we would find that it splits into two parts (rapid and slow). Of particular interest is the viscosity $\eta(q)$ for flow modulated in space at a wavelength $2 \pi / q$. This generalized viscosity is useful for the discussion of melt flow near a solid boundary : i.e. in small tubes, or near a small solid particle in suspension. $\eta(q)$ is related to $\Gamma_{q}(t)$ by a Kubo formula

$$
\eta(q)=\frac{1}{k T} \int_{0}^{\infty} \Gamma_{q}(t) \mathrm{d} t
$$

This will be dominated by the slow component. Using the Doi-Edwards analysis, it is easy to see that

$$
\eta(q) \cong E \bar{T}_{\mathrm{rep}} \quad\left(D^{-1}>q>R_{0}^{-1}\right)
$$

where $E=k T /\left(N_{\mathrm{e}} a^{3}\right)$ is the plateau modulus of the entangled structure. Thus $\eta(q)$ is independent of $q$ for a very wide range of $q$. This means that, for simple shear flow near a solid wall, it is correct to assume that the velocity gradient is constant near the wall (down to distances of order $D$ ). This was implicitly assumed in our discussion of boundary conditions for the flow of polymer melts [12].

Acknowledgments. - I have benefited from very helpful discussions on Brownian motions in tubes with F. Brochard and P. Pincus.

\section{References}

[1] De Gennes, P. G., J. Chem. Phys. 72 (1980) 4756.

[2] Higgins, J. S., Proceed. Conf. on Neutron Scattering, Pt. I Gattinburg, Tenn., 6-10 June 1976, p. 344. Allen, G. and MaconNaChIE, A., Br. Polym. J. 9 (1977) 184.

[3] Van Hove, L., Phys. Rev. 95 (1954) 249.

[4] Richter, D., Hayter, J., Mezei, F., Ewen, R., Phys. Rev. Lett. 42 (1979) 1681.

HAYTER, J., Private communication.

[5] Rouse, P. E., J. Chem. Phys. 21 (1953) 1272.

[6] De Gennes, P. G.. Phivic 3 (1967) 37.
[7] De Gennes, P. G., Dubois-Violette, E., Physics 3 (1967) 181.

[8] De Gennes, P. G., J. Chem. Phys. 55 (1971) 572.

[9] Doi, M., Edwards, S. F., J. C. S. Faraday II 74 (1978) 1789, $1802,1818$.

[10] De Gennes, P. G., C. R. Hebd. Séan. Acad. Sci. (Paris), to be published.

[11] Pincus, P., to be published in J. Chem. Phys.

[12] De Gennes, P. G., C. R. Hebd. Séan. Acad. Sci. (Paris) B 288 (1979) 219. 